\title{
Adenocarcinoma of the Esophagogastric Junction: Territory of the Esophagus or Stomach, or an Independent Region?
}

\author{
Hiroya Takeuchi, MD, PhD and Yuko Kitagawa, MD, PhD, FACS \\ Department of Surgery, Keio University School of Medicine, Tokyo, Japan
}

The most recent union for international cancer control (UICC) tumor-node-metastasis (TNM) classification (7th edition) definitively classified adenocarcinoma of the esophagogastric junction (AEG) for the first time. ${ }^{1}$ A tumor whose epicenter is within $5 \mathrm{~cm}$ of the esophagogastric junction (EGJ) and also extends into the esophagus is classified and staged according to the esophageal cancer scheme. All other gastric tumors with an epicenter greater than $5 \mathrm{~cm}$ from EGJ or within $5 \mathrm{~cm}$ of EGJ without extension into the esophagus are staged using the gastric cancer scheme. Therefore, most AEGs, including adenocarcinomas of the cardia and subcardia, are now to be staged as esophageal adenocarcinomas rather than gastric cancers by the current edition of TNM classification.

AEG has been classified into three subtypes during the last decade, as advocated by Siewert and Stein AEG type I (distal esophageal adenocarcinoma) is a tumor with the epicenter located 1-5 cm above EGJ regardless of invasion to EGJ. ${ }^{2,3}$ AEG type II (true carcinoma of the cardia) is a tumor invading EGJ with the epicenter located between $1 \mathrm{~cm}$ above and $2 \mathrm{~cm}$ below EGJ. AEG type III (carcinoma of the subcardia) is a tumor invading EGJ with the epicenter located $2-5 \mathrm{~cm}$ below EGJ. It is likely that AEG type I tumors may be esophageal adenocarcinomas, which are mainly derived from the Barrett's esophagus. However, whether AEG type II/III tumors should be also classified as esophageal adenocarcinomas still remains unknown.

Recently, Gertler et al. ${ }^{4}$ attempted to evaluate whether AEG type II/III tumors are better staged as esophageal or gastric cancer by analysis of a single-center cohort of 1,141 patients who underwent surgery for AEG type II/III tumors in Germany. In their cohort, by esophageal cancer staging

(C) Society of Surgical Oncology 2012

Published Online: 7 December 2012

H. Takeuchi, MD, PhD

e-mail: htakeuchi@a6.keio.jp and gastric cancer staging as per the 7th edition UICC TNM classification, the authors emphasized that some stage groups were lacking homogeneity (absence of distinct subsets of patients within a stage), monotonicity (continuous decreasing survival with increasing stage group), and distinctiveness (difference in survival between monotonic stage groups). ${ }^{4}$ They concluded that neither the esophageal nor the gastric scheme was perfectly suitable for patients with AEG types II/III in terms of prognostic stratification. In contrast, Huang et al. ${ }^{5}$ analyzed 142 consecutive resection patients in a single Chinese center and suggested that it may be better to classify carcinomas of the cardia involving the esophagus using gastric cancer staging than esophageal cancer staging of the 7 th edition TNM classification.

Hasegawa et al. reported the postoperative survival of 163 patients with AEG types II/III when classified by either the esophageal cancer scheme or gastric cancer scheme of the 7 th edition TNM classification. ${ }^{6}$ When the esophageal cancer scheme was used, patient distribution was substantially deviated to stage IIIC and the survival curves for each stage were not well separated. In contrast, when the gastric cancer schema was used, patient distribution was relatively even and the survival curves were monotonously and distinctively separated. They concluded that gastric cancer staging may be more suitable than esophageal cancer staging for patients with AEG types II/III. These results should be interpreted in context with caution because they represent a single-center retrospective cohort study with a small number of patients. Nevertheless, that study may provide a valuable signpost for the future direction of the AEG staging system.

Hulscher et al. reported no significant difference in the postoperative survival between transhiatal esophagectomy (THE) and transthoracic esophagectomy (TTE) in patients with distal esophageal adenocarcinoma (AEG type I) or gastric adenocarcinoma of the cardia involving the distal 
esophagus (AEG type II) in a prospective randomized phase III trial. ${ }^{7}$ TTE with extended lymphadenectomy was associated with higher morbidity than THE; however, subgroup analysis after long-term follow-up recently demonstrated that patients with 1-8 positive lymph nodes significantly benefited from extended transthoracic lymphadenectomy in the 5-year locoregional disease-free survival and overall survival. ${ }^{8}$ Furthermore, transthoracic resection resulted in a $14 \%$ 5-year survival benefit (37\% after THE vs $51 \%$ after TTE) for patients with esophageal cancer (AEG type I), although the difference was negligible (31\% after THE vs $27 \%$ after TTE) for patients with cancer of the cardia (AEG type II). Sasako et al. reported the results of a phase III trial that revealed no significant difference in the postoperative overall survival between transhiatal and left thoracoabdominal approaches in patients with gastric adenocarcinoma of the cardia or subcardia (AEG type II/III) with esophageal invasion of $3 \mathrm{~cm}$ or less. ${ }^{9}$ These results suggest that AEG type I may be classified as esophageal adenocarcinoma and AEG type II/III as gastric cancer not only for better prognostic stratification but also for appropriate selection of surgical procedures. The extent of lymphadenectomy in patients with AEG would be optimized according to the location of the primary tumor, any metastatic lymph nodes, and individual lymphatic flows.

We recognize that additional evidence based on large multicenter prospective studies is required. Studies evaluating the postoperative survival of patients with AEG are required to clarify whether tumor types I, II, and III should be classified as esophageal adenocarcinomas or gastric cancers. We hope that AEG tumors will find their suitable territories (esophagus, stomach, or an independent region).
Appropriate classification will help to provide promising surgical strategies and standardized management for patients with AEG.

\section{REFERENCES}

1. UICC. Oesophagus including oesophagogastric junction. In: Sobin LH, Gospodarowicz M, Wittekind C, editors. TNM classification of malignant tumours. 7th ed. New York: Wiley; 2009. p. 66-72.

2. Siewert JR, Stein HJ. Classification of adenocarcinoma of the oesophagogastric junction. Br J Surg. 1998;85:1457-9.

3. Stein HJ, Siewert JR. Cancer of the esophagogastric junction. Surg Oncol. 2000;9:35-41.

4. Gertler R, Stein HJ, Loos M, Langer R, Friess H, Feith M. How to classify adenocarcinomas of the esophagogastric junction: as esophageal or gastric cancer? Am J Surg Pathol. 2011;10:1512-22.

5. Huang Q, Shi J, Feng A, Fan X, Zhang L, Mashimo H, et al. Gastric cardiac carcinomas involving the esophagus are more adequately staged as gastric cancers by the 7th edition of the American Joint Commission on Cancer Staging System. Mod Pathol. 2011;24:138-46.

6. Hasegawa S, Yoshikawa T, Aoyama T, et al. Esophagus or stomach? The seventh TNM classification for Siewert type II/III junctional adenocarcinoma. Ann Surg Oncol. 2012. doi:10.1245/ s10434-012-2780-x.

7. Hulscher JB, van Sandick JW, de Boer AG, Wijnhoven BP, Tijssen JG, Fockens P, et al. Extended transthoracic resection compared with limited transhiatal resection for adenocarcinoma of the esophagus. N Engl J Med. 2002;347:1662-1669.

8. Omloo JM, Lagarde SM, Hulscher JB, Reitsma JB, Fockens P, van Dekken H, et al. Extended transthoracic resection compared with limited transhiatal resection for adenocarcinoma of the mid/distal esophagus: five-year survival of a randomized clinical trial. Ann Surg. 2007;246:992-1001.

9. Sasako M, Sano T, Yamamoto S, Sairenji M, Arai K, Kinoshita T, et al. Left thoracoabdominal approach versus abdominal-transhiatal approach for gastric cancer of the cardia or subcardia: a randomised controlled trial. Lancet Oncol. 2006;7:644-51. 